\title{
Microscopic Analysis of Chips
}

\author{
Dominik Malcik, Martin Drahansky \\ UITS, FIT BUT \\ imalcik@fit.vutbr.cz,drahan@fit.vutbr.cz.
}

\begin{abstract}
Nowadays many different types of chips are used virtually everywhere in the real world. Sometimes, it is necessary to ensure that a certain chip meets specific requirements. For this reason, it is essential to examine various properties of chips; one of those can be, e.g., the chip security with respect to its physical structure.

This paper contains an overview of methods for processing of chips - concretely: chip decapsulation, chip deprocessing, scanning of layers, microscopic analysis and image data analysis.
\end{abstract}

Keywords: chip, analysis, microscopic, microscope, decapsulation, deprocessing.

\section{Introduction}

The complexity of manufactured integrated circuits (IC) is rising rapidly (almost according to Moore's law [1]). Therefore, a performance of a complex analysis can be very difficult and long-winded (especially with no information about the circuit). That is why it is recommended (if at all possible) to only focus on specific parts and leave out the irrelevant ones. The best situation would be if there was a very precisely given location of interest at the beginning of the investigation process. Unfortunately, the area of interest can usually be narrowed down only after a certain effort. Nonetheless, the complex analysis is sometimes plainly required. [2]

A comprehensive analysis contains a software analysis and a hardware analysis. A combination of these two approaches usually leads to a very complex work with a potential to reveal different types of errors or vulnerabilities of the device. It should also be treated as a fact that each type of analysis has to be taken as a standalone discipline. The feasibility of the software analysis is usually much better because of availability of affordable equipment. Nevertheless, in this paper we will cover only parts related to hardware analysis.

\section{Obtaining Chips}

First step of our work is to obtain chips. In our case, the chips are placed in plastic cards.

\subsection{Extraction from Plastic Cards}

Decapsulation of chips in plastic package begins with extraction from plastic cards. First, we cut out the parts with chips inside from the rest of the plastic cards (see Figure 1).

Then, we continued with extraction of the encapsulated chips from the plastic cover. In early stages we used only acetone bath in a beaker at normal room temperature. The extraction took several minutes, or even tens of minutes. The plastic cover was becoming pliable and it was possible to peel the plastic layers off one after another. 


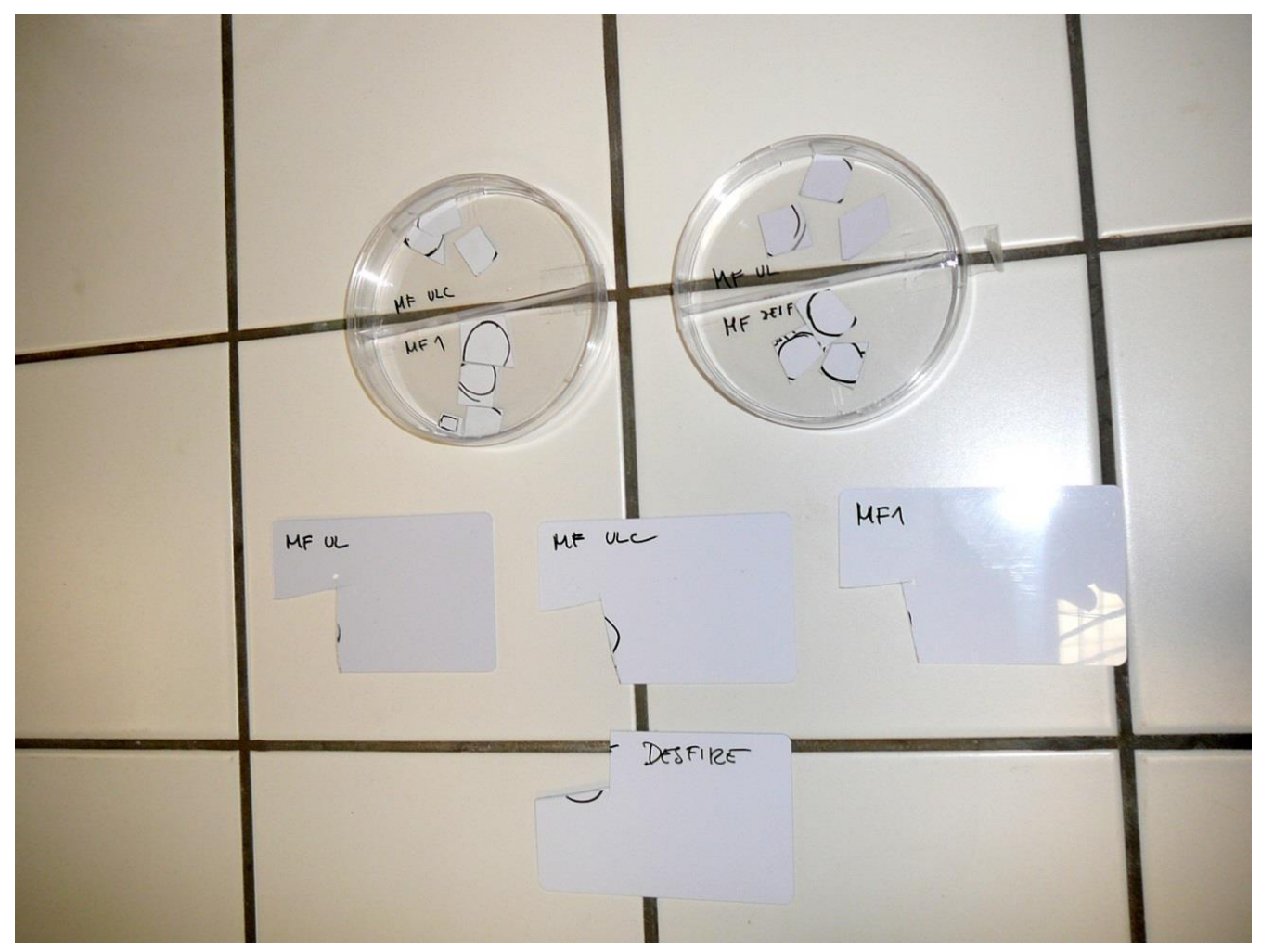

Figure 1. Chips in Plastic Covers

This process was enhanced with use of a boiling nest (displayed on Figure 2). The time of extraction got shortened to 1-3 minutes per piece. We simply boiled the acetone to its boiling temperature (slightly above $50{ }^{\circ} \mathrm{C}$ ), the plastic compound was then almost immediately removable. We highly recommend to use plastic gloves for extraction of the chips because of two reasons:

- The chips might be relatively hot and the plastic gloves help to mitigate the heat interaction between fingers and the chips.

- The plastic compound becomes sticky after the boiling process and it is difficult to clean it from fingers afterwards.

Out of these two points, the first one represents also the only drawback of this improvement - the temperature of boiling acetone is higher than the normal room temperatures. This might cause damage to some chips. Fortunately, the boiling temperature of acetone is not very high and the damage of the chips is therefore not very likely. Nevertheless, this aspect has to be kept in mind, especially in cases when the analyst wants to prevent any harm to the chip. And even more, in cases when it is not known what kind of chip (with what features) is taken under investigation.

\section{Chip Decapsulation}

After obtaining the chip, the next step is to remove the chip packaging. For this purpose, two main approaches are commonly presented - etching and grinding or sometimes polishing instead of grinding. Both are specific and it is always felicitous to have the capability of performing both.

There are several books dealing especially with the chip packages and the related topics, e.g. [8-11]. It is a very wide area, with many important aspects of knowledge. For 
our work it is important to deal with the information regarding managed package destruction - the decapsulation.

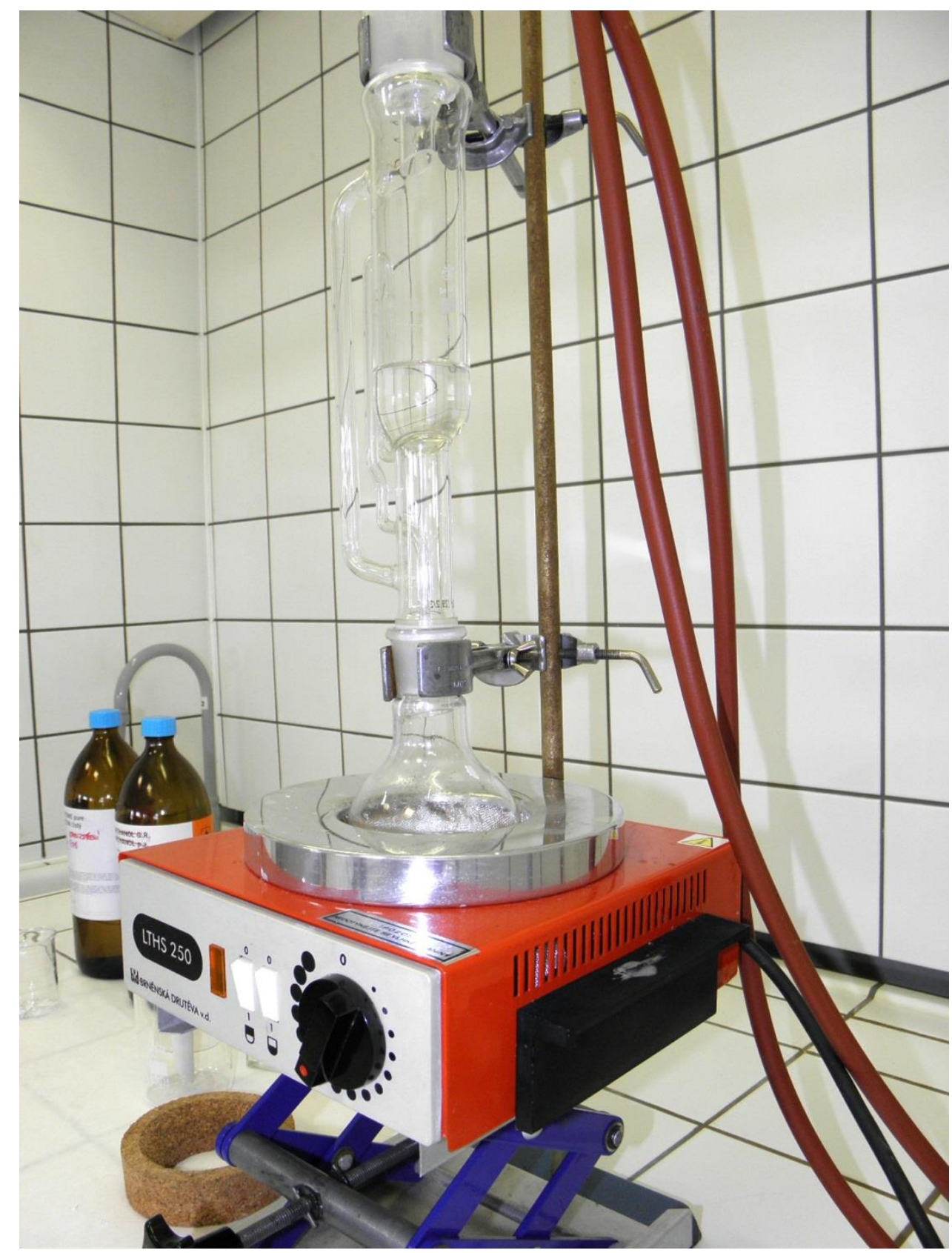

Figure 2. Boiling Nest with Boiling Acetone Bath

It can be said, very briefly, that there are three main types of common chip packages considering their material - metal, ceramic and plastic. The most important category for us is the plastic one. These packages are widely used and are often suitable for most ordinary integrated circuits, at a low price. The metal and ceramic packages do not occur as often as the plastic ones.

We decided to follow the main stream packages - plastic packages. Up to now all the investigated chip packages appertain to this group of packages. The effective approach of obtaining a bare chip out of a plastic package with the use of sulphuric acid and nitric acid 
was performed within the scope of the Brno University of Technology in cooperation with Faculty of Chemistry at Brno University of Technology.

\subsection{Chemical Approach}

The chemical approach is usually more convenient, because of simplicity, low time requirements, low price and availability of the chemicals involved. The fact is that the chemicals are chosen in a manner that they react only with specific compounds - the danger of damaging the sample surface is then minimal. Different variants of the chemical approach make use of the same acids, but mostly in a more or less different manner [3]. However, some of them are totally dissimilar. Another technique is based on using resin instead of acids (briefly described in [4]). However, to achieve the desired results, it takes a lot of time. And in fact, it is not recommended to follow this approach because resin vapor can foul up an exhaust. Moreover, the temperature of resin during the etching process should be really high, ca. $350{ }^{\circ} \mathrm{C}$. This temperature may cause a serious damage to a chip. On the other hand, the use of dangerous acids is unnecessary.

Therefore, the process can practically be performed somewhere outside a building to avoid a necessity of using any exhaust at all. In that case, the work can be done without a proper chemical laboratory. Considering the price of resin and the fact that storing resin is safe, this approach may sometimes be more convenient. [5][6][7]

Plastic packages differ one from another. Due to this fact, a certain package provides unique features suitable for a particular purpose. However, the composition is usually considerably similar and that is why the same process can be used to remove almost all types of such molding compounds - the described process should be taken as a template because the diversity of the plastic compounds requires certain flexibility in performing the procedure. Let us assume that all the next steps take place in a chemical laboratory properly equipped for the etching process. At least a fume cupboard, a chemical sink and personal protection equipment should be available.

\subsubsection{Required Equipment}

This part provides a list of items needed to complete the process of decapsulation. Without these items we cannot recommend to perform the described procedure, because of potential danger - in a better case the samples may be destroyed, in the worst case scenario, serious injury may occur.

- Sulphuric acid (H2SO4 - concentration $96 \%$ or more);

- Nitric acid (HNO3 - concentration $96 \%$ or more);

- Acetone (C3H6O);

- Demineralized water or distilled water (in the worst case tap water);

- Ultrasonic cleaner;

- Cooker with fume cupboard;

- Beakers (at least four pieces);

- Tweezers;

- Filter paper or nitrogen flow;

- Personal protection equipment (gloves, glasses, protective clothes etc.);

- Microscope or magnifier;

- Box to place the chips in.

In some situations it is convenient to use an X-ray to get some more information about the chip inside the package prior to the decapsulation process. 


\subsubsection{Ratio of Nitric Acid to Sulphuric Acid}

Before we describe the process, let us introduce a very important part. Prior to beginning the etching itself it has to be decided what is expected as a result of the etching. According to our needs, the correct ratio of the acids mixture has to be chosen. The result is affected not only by the acids ratio, but also by the time duration of the active etching (the time period when the specimen is inside the acid bath) and also by the temperature of the acids.

It has to be kept in mind that the compounds can be significantly dissimilar. This fact can cause the time of etching to differ significantly as well. We experienced the etching duration from tens of seconds to tens of minutes (the longest etching was performed on a type of RFID chips - about 40 minutes).

The ratio is always stated as nitric acid to sulphuric acid (the recommended approx. temperature is mentioned in brackets).

- $5: 1-3: 1$ (ca. $90-94{ }^{\circ} \mathrm{C}$ ) - preservation of wire bonds from the leadframe to the chip.

- 2:1 - $1: 1$ (ca. $90-94{ }^{\circ} \mathrm{C}$ ) - faster, cheaper and a more aggressive decapsulation.

- 0 : 1 (up to ca. $270{ }^{\circ} \mathrm{C}$ ) - to etch very resistant molding compounds, very aggressive.

\subsubsection{Whole-package Decapsulation Process}

It is strongly recommended to proceed very cautiously while observing the chemical laboratory rules to avoid any injuries or damage to the laboratory equipment or to the chips.

First, four beakers should be prepared. Two beakers should be half-filled with the acids in a correct ratio (see above). The third beaker should be half-filled with acetone and the last one with demineralized water. The cooker must be placed into a fume cupboard because of the production of dangerous vapors. Only the beakers with acids need to be placed on the cooker in order to reach the desired working temperature. The other steps of the whole process should take place out of the cooker.

The major etching should be performed in the first beaker with the acids. The acids will become non-transparent soon due to the presence of etched molding compounds. The recommendation is to periodically inspect the level of decapsulation. When the process is almost complete, it is better to use the second beaker with the acids to do the fine etching. It is necessary to monitor the progress permanently at this point. Transparent acid is ideal for this purpose. We do not recommend leaving the specimens in the acid bath longer than it is necessary - the lower layers could be damaged by the acid because these layers are usually not protected along the chip edges.

When the chip is bared, it should be washed in the beaker with acetone, then with flowing demineralized water and it should be put inside another beaker with demineralized water afterwards. In the case where the chip surface is bigger than $5 \mathrm{~mm}^{2}$, we recommend using demineralized water, acetone and demineralized water again due to safety reasons - a bigger amount of the acid left on the chip can react with acetone.

The beaker with the demineralized water and a bared chip or chips, as the case may be, should be placed into an ultrasonic cleaner for up to two minutes in the case where there is only one chip in the beaker or for ca. thirty seconds in the case where there are more chips in the beaker (with a little exaggeration, the chips act as emery paper). Then a final check should take place. If everything seems to be alright, the chips should be dried with filter paper or nitrogen flow. The nitrogen flow has to be used very cautiously because the chips are lightweight and it is very difficult to find them on the floor, especially the smaller ones. Then the chips should be properly stored. If there is any problem with the cleanliness of the chip surface or similar, the entire process can be repeated. 


\subsubsection{Etching of a Specific Packaging Part}

A useful approach is to only etch a specific part of the chip package. The entire chip is preserved and, hence, it can be attached to other components as usual. This is needed when some measurements have to occur, or only when it is necessary to preserve the whole structure of the chip. The chip surface can still be observed via the etched aperture. Manufacturers use this method relatively often to perform specific checks of the chip surface or of the wire bonds connections, etc.

Because of the manual dosing of acid drops to the specific area with a dropper, this process is more demanding. First, a little hollow is made in the surface of the chip package (approx. in the center of the area designated for etching). Then, the drops of sulphuric acid are manually applied to the chosen location (usually, nitric acid is not used in this approach). There has to be a short period of inactivity between the applications of the acid drops to allow reaction with the surface. It is undesirable to apply more acid than is imperative, because the reaction is not under perfect control in that case, and therefore, the result cannot be predicted or even guaranteed.

\subsection{Grinding and Polishing}

The grinding and polishing approach is suitable in cases where we cannot apply the chemicals, or the chip package allows the use of a grinder with its advantages, e.g. the chip is placed deeper in the package and there is a gap above the chip - in that case the wirebonds can be easily preserved. However, it is necessary to proceed cautiously to avoid contact of the grinder with the surface, or wirebonds, of the chip - for this reason it is recommended to use an X-ray to inspect the starting situation and then a current level of decapsulation [5-7].

\subsection{Decapsulation in Practice}

Now we got to a point where we have chips in their original plastic packages placed on a metal pad - see Figure 3. The package is in fact the last plastic layer that separates the circuits from the outer world. 


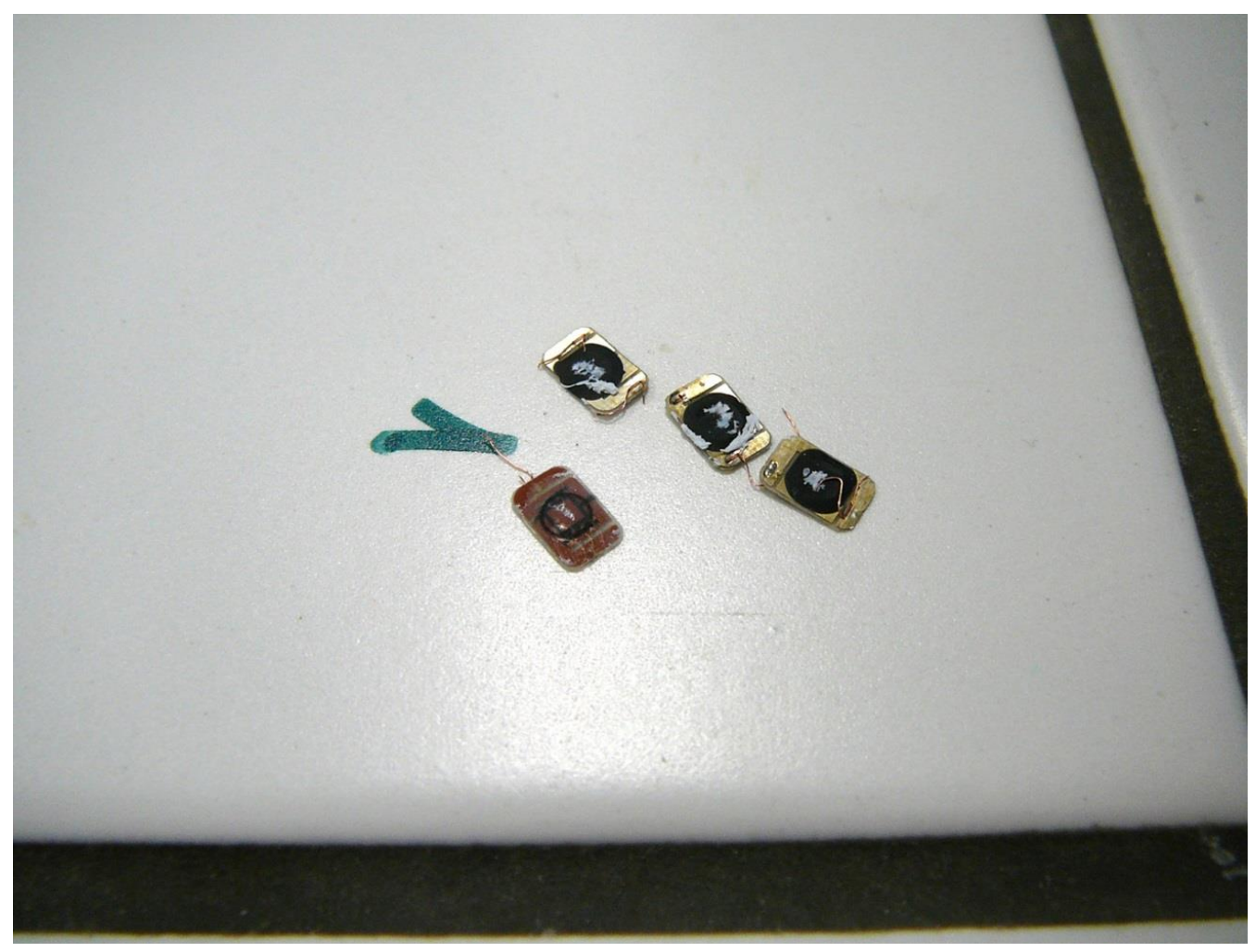

Figure 3. Chips in Plastic Packages on Metal Pads

Since we knew that the chips were resistant against damage of the surface by sulfuric acid - because of the top level passivation layer - we decided to use only the sulfuric acid to speed up the decapsulation process. Furthermore, work with only sulfuric acid is more comfortable and also safer than work with the proposed mixture of sulfuric and nitric acids.

In a normal case, we would have to rather proceed more cautiously during the decapsultaion. In this case we had done several attempts of the decapsulation with these chips before. That is why we already knew that the chips were resistant against the sulfuric acid damages and this knowledge allowed us to perform the faster way of decapsulation process with use of only the more aggressive acid.

The Figure 4 shows the sulfuric acid bath, on the left there is the beginning of the whole process. The beakers are labelled with numbers; number 1 is used for the rough decapsulation. The acid in it will become less transparent soon after the beginning of the process. That is why, the final part of the decapsultaion process is carried out in the second beaker with acid, labelled as number 2 . In the second beaker with fully transparent acid it is much easier to determine the end of the process. Leaving chips too long in the hot acid bath might cause their complete destruction, no matter how resistive they are.

After the successful decapsultaion, we finally get the chips for the next step, the decomposition, sometimes called also delayering. 


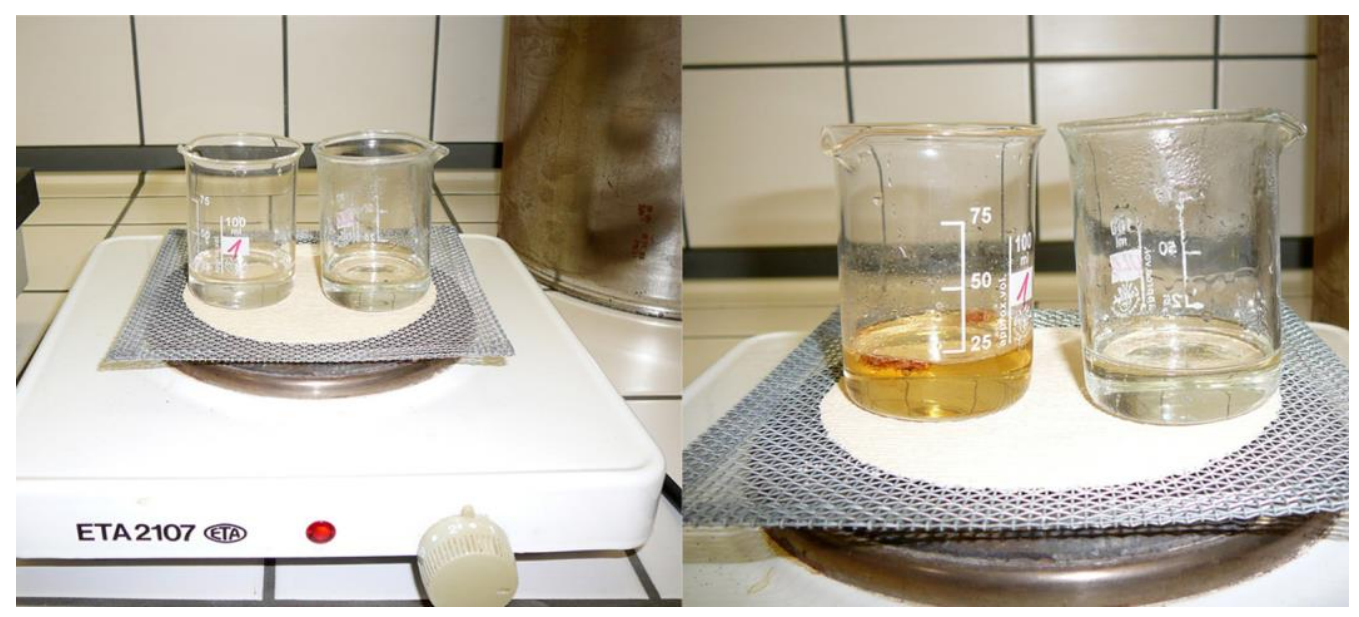

Figure 4. Chips in Acid Bath. On the Left: fresh bath; on the right: bath colored from the melting plastic packages

\section{Chip Delayering}

The chip deprocessing (delayering) involves three main techniques: dry etching (plasma etching), wet etching using different types of chemicals and polishing. Each particular type of chip is unique because of composition of layers and used materials. That is why, an appropriate procedure has to be prepared according to the real conditions regarding each type of chip. In some situations, it is possible to simply assume a common composition and materials, but the diversity of materials and compounds is growing day by day. The best practice is to start with a cross-section analysis including a layers thickness measurement and materials analysis. Only then the correct procedure can be created and performed.

Although a suitable chemical procedure can usually be found for each layer, sometimes it is not possible to choose wet etching because of the high risk of damaging other layers. In these cases it is still possible to make progress with a grinder and a decent level of skillfulness.

One basic principle is valid for all types of procedures in this sphere - it is necessary to inspect the current progress periodically; and very often. The structures are miniature indeed - the progress of employed technique can be faster than we could ever imagine.

\subsection{Deprocessing of Common Layers}

According to the information given earlier in this text, the decomposition process usually consists of a specific sequence of particular steps.

\subsubsection{Passivation}

The very first layer is mostly a passivation - a kind of chip protection. To remove this layer, it is recommended to use plasma etching. The whole process takes about 45 minutes with an old plasma etcher TESLA 214 VT. The actual plasma etching lasts only 4 minutes out of the mentioned time period. The length of plasma etching depends on the type of plasma etcher and should be adjusted according to the particular machine performance. The rest of the time is devoted to preparing conditions necessary for performing this procedure.

\subsubsection{Aluminum Compounds}

Conductive layers made of aluminum compounds can be taken away by application of phosphoric acid etching mixture, PEWS 765-140-57-36 3. The recommended working 
temperature is around $50{ }^{\circ} \mathrm{C}$, the common time of bath should be from 2 to 6 minutes depending on the layer thickness.

\subsubsection{Oxide Compounds}

A special chemical mixture is also available for removing oxide compounds insulating material. Precisely, the mixture consists of ammonium fluoride and hydrofluoric acid in ratio 7:1. The working temperature is $30^{\circ} \mathrm{C}$. The common time of bath should be from 2 to 6 minutes.

\subsection{Deprocessing in Practice}

Decomposition of the chips should start with a cross-section - see Figure 5. Crosssection reveals the number of layers, their thickness and some of the interconnections. The subsequent main delayering is then much easier.

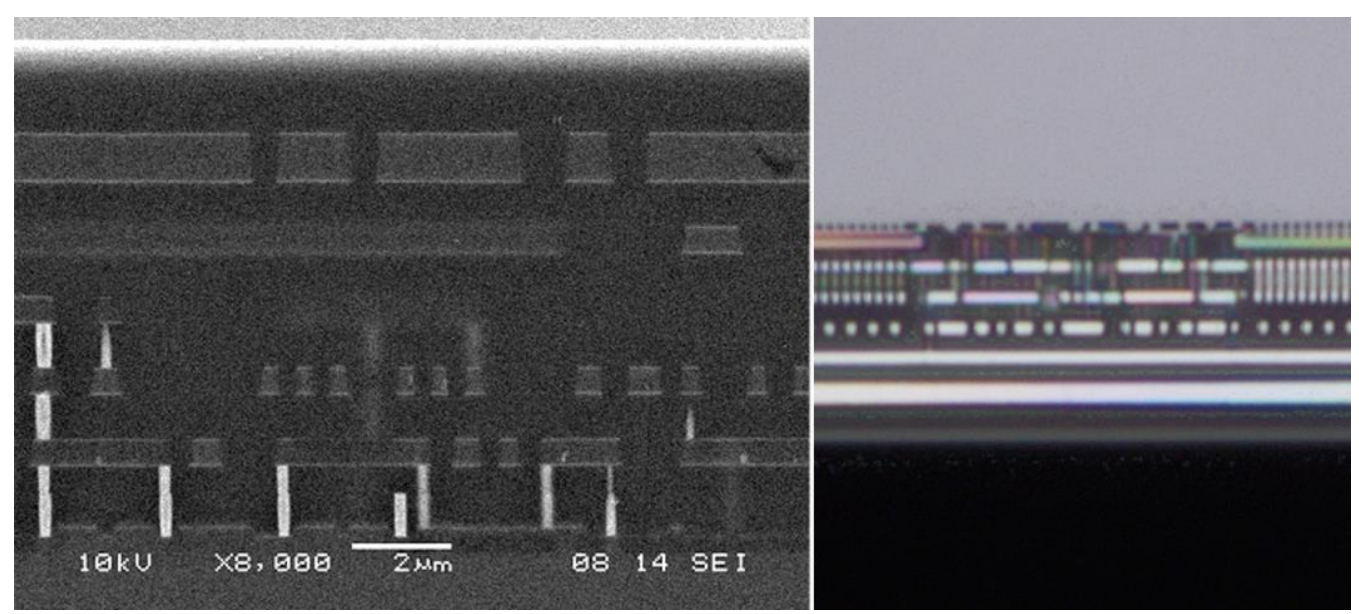

\section{Figure 5. On the Left: Cross-section of a Chip Acquired by an Electron Microscope; on the Right: the Same Chip Cross-section Acquired by an Optical Microscope}

In our case, we did the cross-section only for one type of chips and the others were deprocessed experimentally - the results of experimental delayering of Mifare Classic chip are displayed in figures Figure 6, Figure 7, Figure 8 and Figure 9. We had enough samples and the experimental approach is usually sufficient, unless we want to use grinder to remove layers by polishing. 

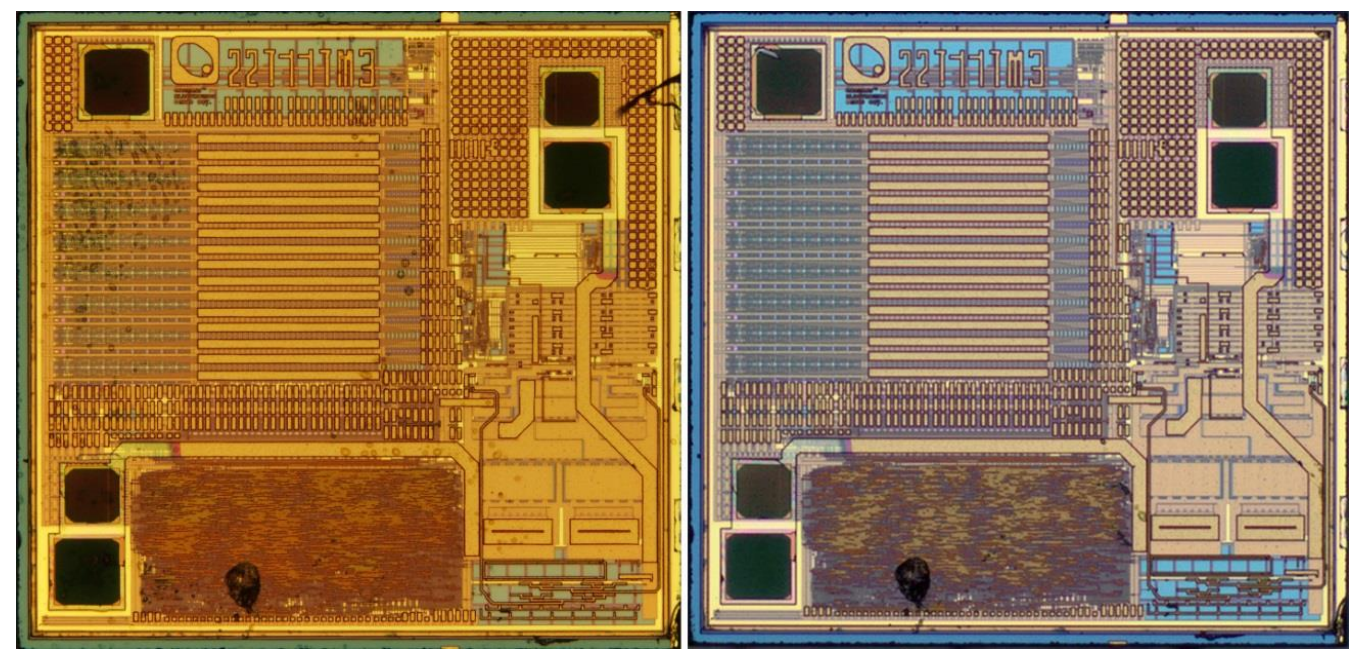

Figure 6. Mifare Classic Delayering - on the Left: Chip with Passivation Layer; on the Right: Chip with Removed Passivation

Due to the fact that we always used chemicals instead of polishing method, we simply relied on the assumption that we can expect a conductive layer after a passivation layer. And after each conductive layer, there should be a layer of an insulant. The compounds used commonly for the particular types of layers are known and that is why we could use chemicals according to our expectations. In a case that the order of layers would be different from our assumptions, there should be either no reaction at all or a very small reaction seen mainly on edges of the chip, because of underetching of the top level layer.
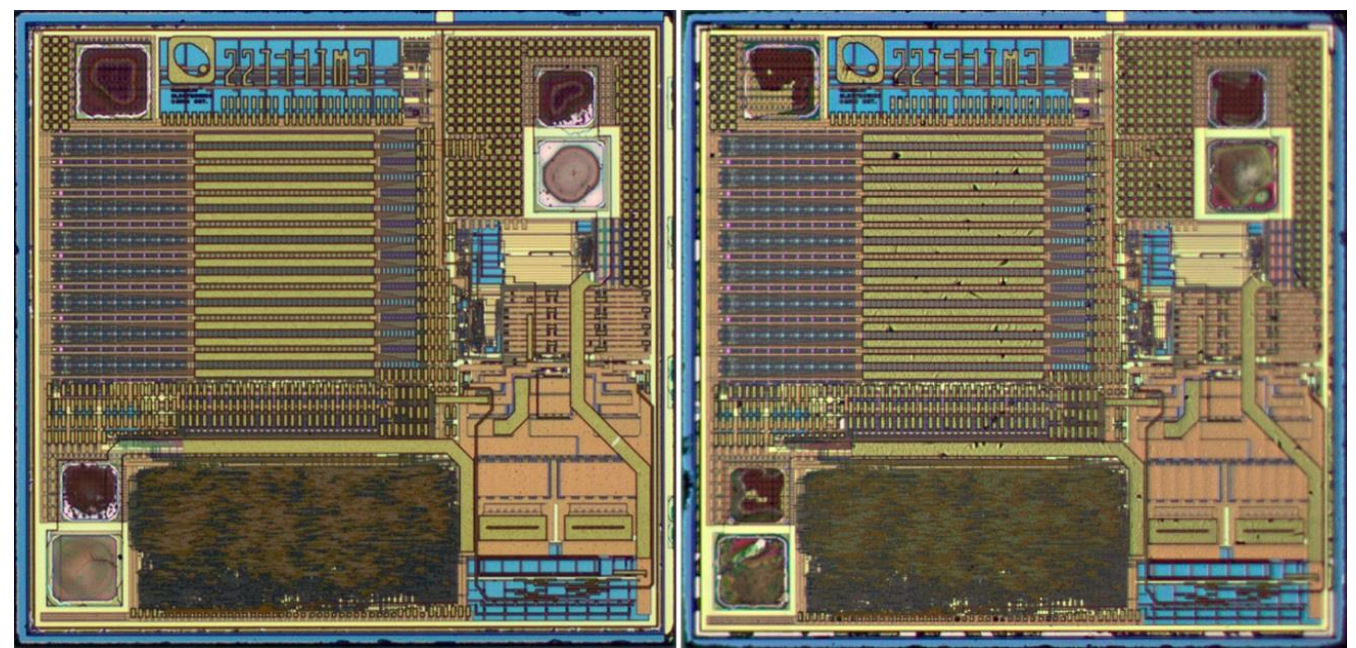

Figure 7. Mifare Classic delayering - on the Left: Chip with Removed Passivation and First Metal Layer; on the Right: Chip with Removed Passivation, First Metal Layer and First Insulant Layer 

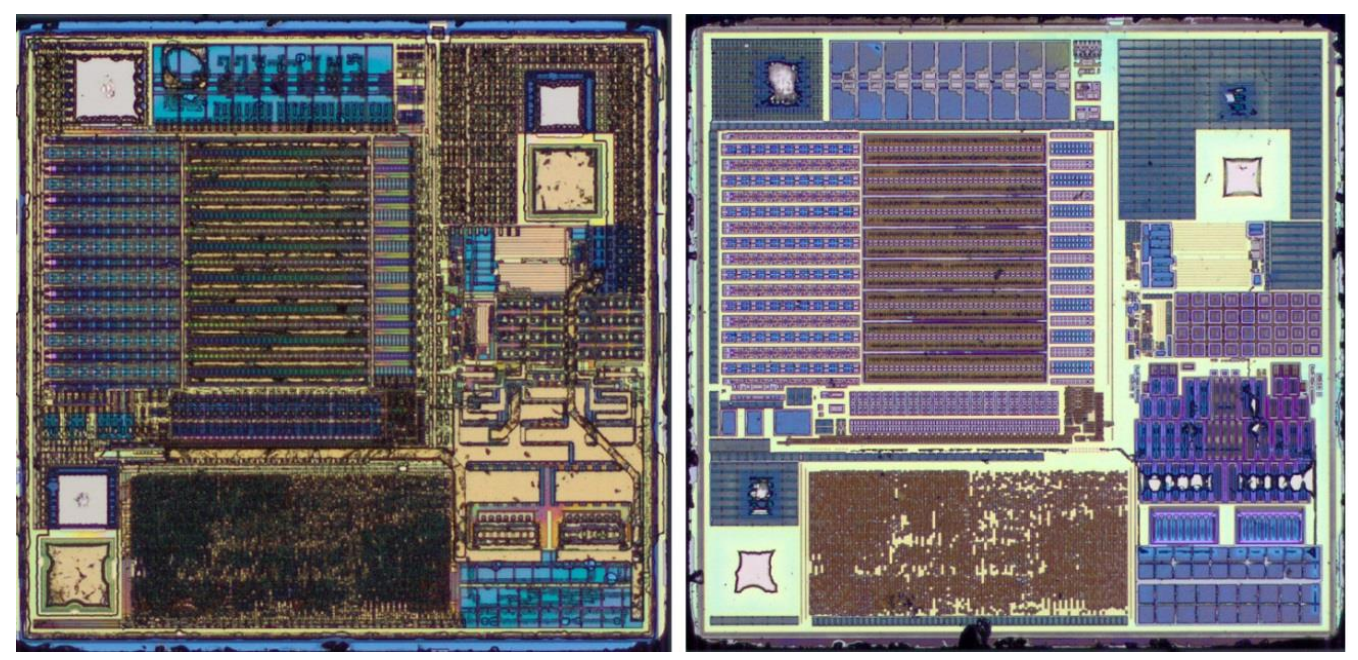

Figure 8. Mifare Classic Delayering - the Next two Steps of Delayering, Oxide Compounds and Conductive Compounds Removing, Alternately

The reaction of chosen chemicals with every unknown layer was tested first. The test was carried out with one sample and for very limited time. This short time test of interaction between the current top layer and chemicals shows whether there is the expected reaction or not.

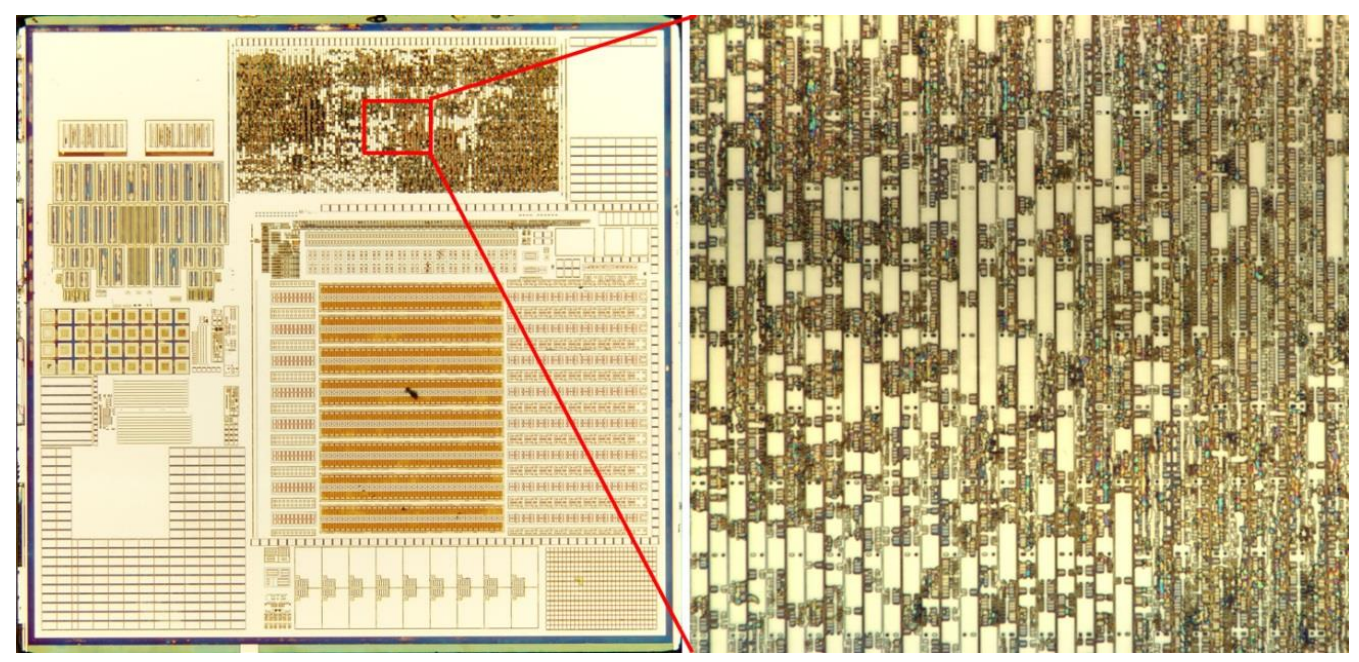

Figure 9. Mifare Classic Delayering - on the Left: Semiconductor Surface; on the Right: Transistor Part Zoomed in with an Optical Microscope

\section{Scanning of the Chips}

All the scanning procedures of deprocessed chips were performed at the Faculty of Information Technology with use of optical microscope Olympus BX61 and electron microscope Phenom Pure G2 - see Figure 10. Only the cross-section pictures and inspectional images necessary for further processing by performing delayering were taken in our partner's laboratories in Onsemiconductor, Rožnov pod Radhoštem. Current images can be considered as a result of experiments in decapsulation and deprocessing. 

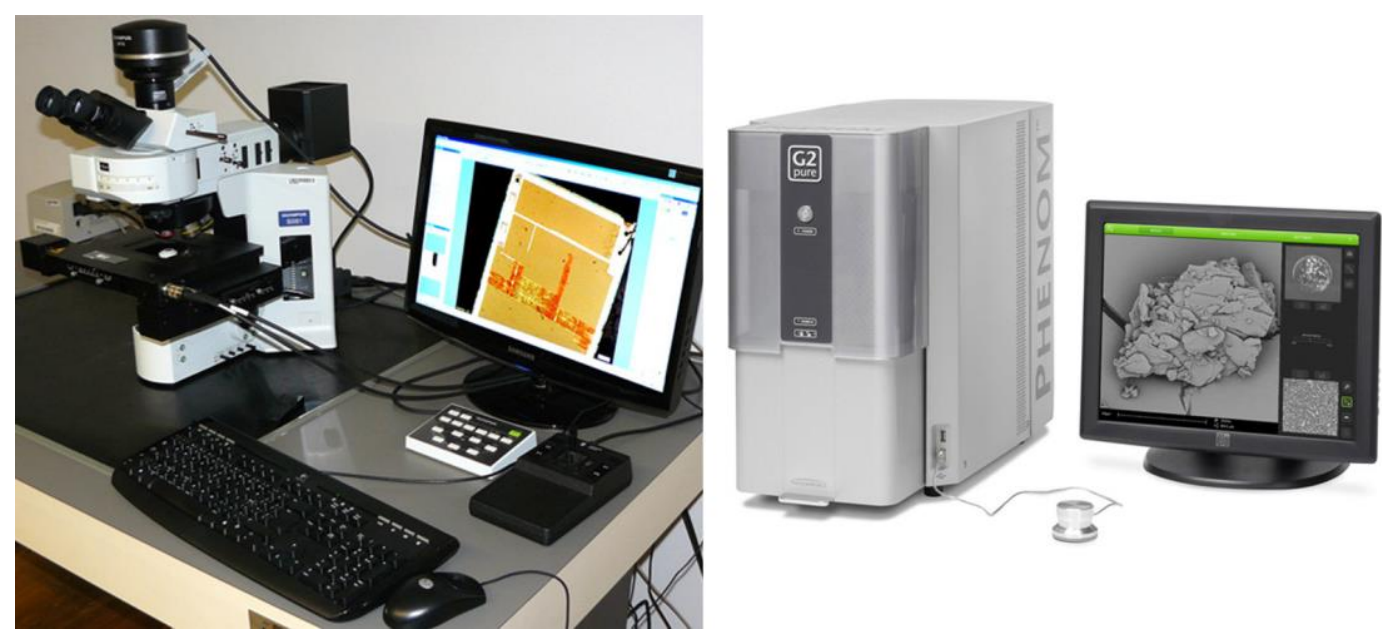

Figure 10. On the Left: Optical Microscope Olympus BX61; on the Right: Electron Microscope Phenom Pure G2

Scanning with both microscopes was done manually. The scheme of scanning was the same for both types of microscopes - acquiring tiles one by one by hand, going across the whole surface in a snake style (see Figure 11). The density of tiles depends, of course, on the magnification. And the magnification depends on the technology used for the observations. We had to take into account also the necessary overlapping of the tiles for later manual/semi-automatic tilessewing.

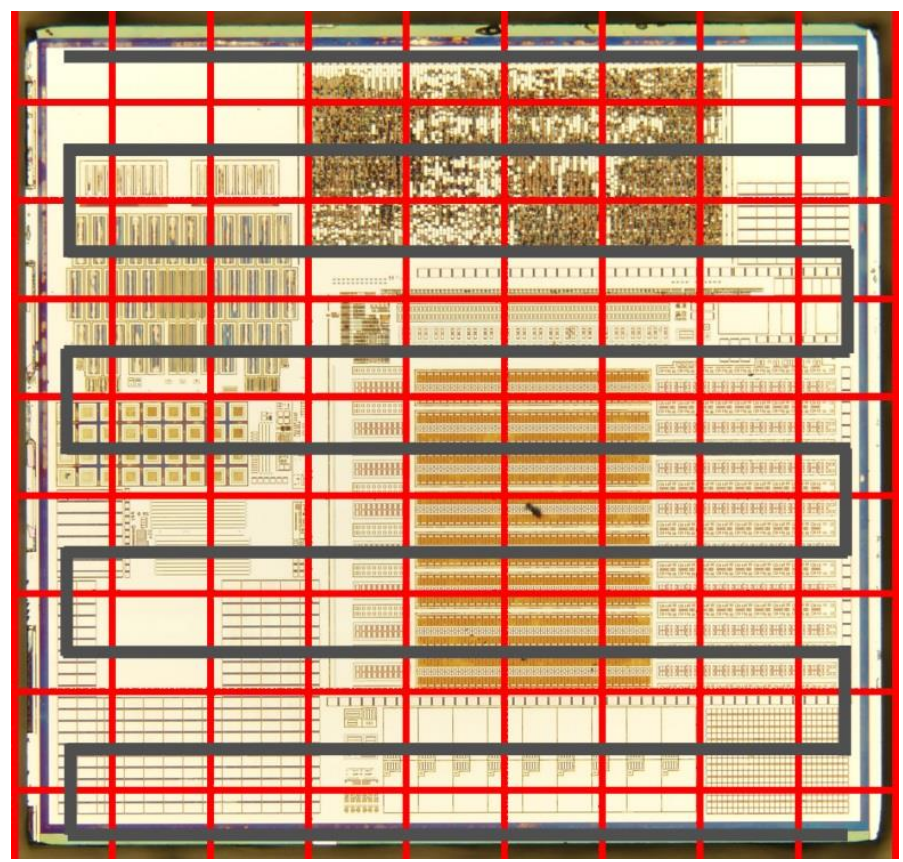

Figure 11. On the Left: Optical Microscope Olympus BX61; on the Right: Electron Microscope Phenom Pure G2

\subsection{Scanning with Optical Microscope}

The optical microscope is equipped with a function of automatic tiles scanning and subsequent tiles sewing. This function is, unfortunately, not reliable and not usable in practice. 
Optical microscopes also suffer from a problem of non-uniform focus within a single image. This problem occurs because of the used technology. System of lenses cannot auto-handle improper height positioning of the specimen on the laboratory glass. Therefore, we had to acquire each tile several times with different focus settings to be able to merge more images into one to get proper focusing across the whole tile.

This manual work is time consuming, e.g., a small chip Mifare Classic consumes two to three hours of manual scanning per each layer without post-processing (merging images to get proper focus, sewing tiles). A complete processing of the whole chip in this way takes more than 5 days, but only in case of a very efficient scanning with no errors encountered in later phases of processing.

It is, therefore, recommended to focus only on a specific part of the chip that might be contributive and relevant for achieving of the required goals.

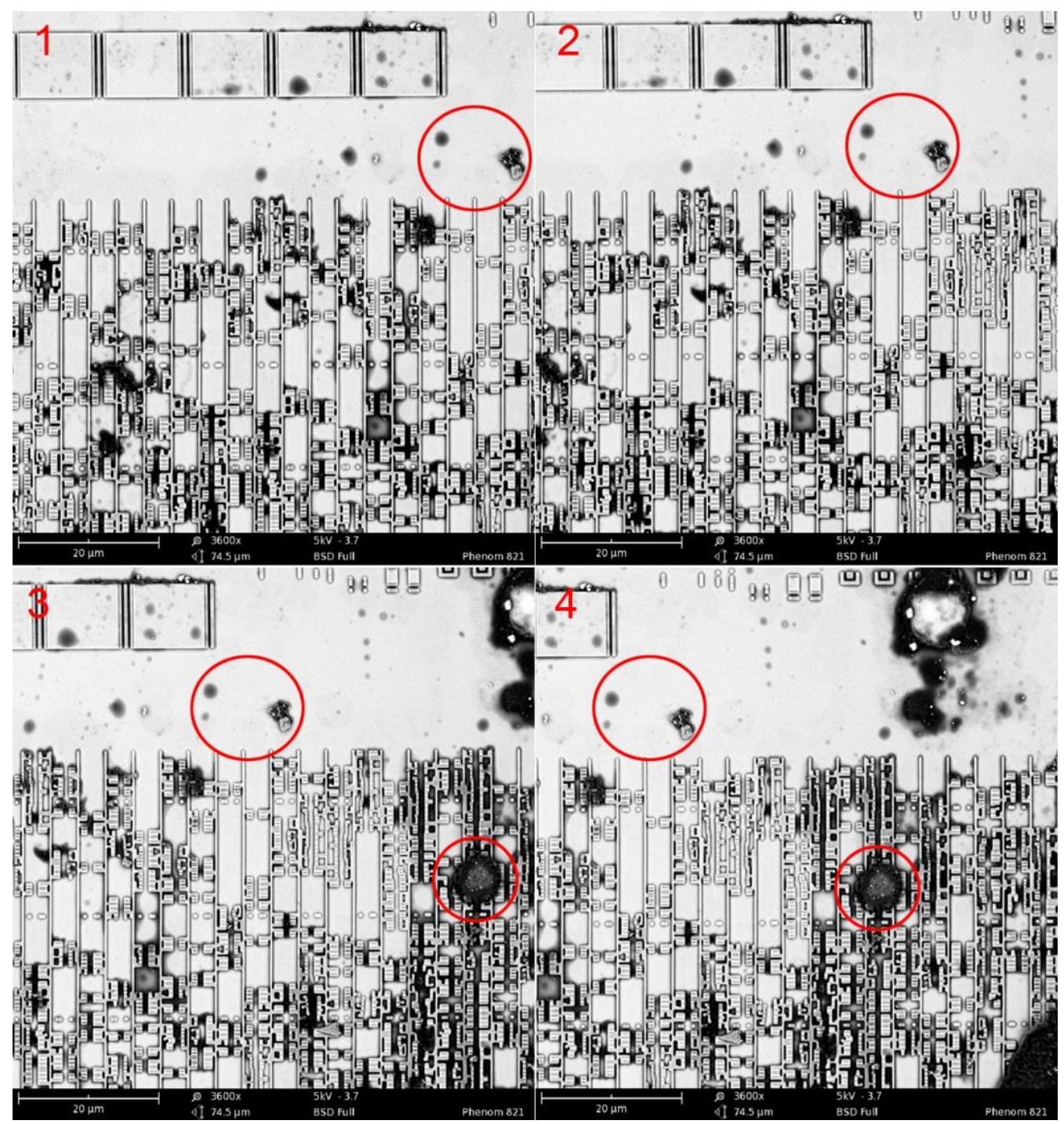

Figure 12. Illustration of the Manual Scanning Scheme Performed with the Electron Microscope; Parts Representing Degree of Overlapping are Marked with Red Circles 


\subsection{Scanning with Electron Microscope}

It can be stated, according to the experience with the optical microscope, that it is virtually impossible to manually scan the whole surface of a chip with respect to its size, possible magnifications provided by electron microscopes and amount of layers.

On the other hand, the problem with focus does not appear with electron microscopes. Every single point of an image is focused. That is undoubtedly a big advantage of this technology.

With our electron microscope, we focused mainly on the semiconductor layer on the areas with transistors. The data is usually stored in tiles with resolution 1024 x 1024 pixels in jpeg format. The highest resolution that Phenom Pure G2 can perform is $2048 \mathrm{x}$ 2048 pixels per image, unfortunately the speed of acquisition with the highest resolution is not suitable for sequential scanning - see Figure 12. That is why the majority of images is stored only in the resolution 1024 x 1024. Format jpeg was chosen above bmp because of sufficient quality, better disk space utilization and storing speed. The size of data is also influenced by the resolution of the images and by the fact that the electron microscope provides data only in greyscale.

In Figure 13 we tried to illustrate the maximum usable magnification of our electron microscope Phenom Pure G2. As it can be seen, the image is slightly blurry, nevertheless still observable very well. The electron microscope that is available in our laboratory belongs to the basic ones; results can be much better with a professional one from the magnification and also image quality perspectives.

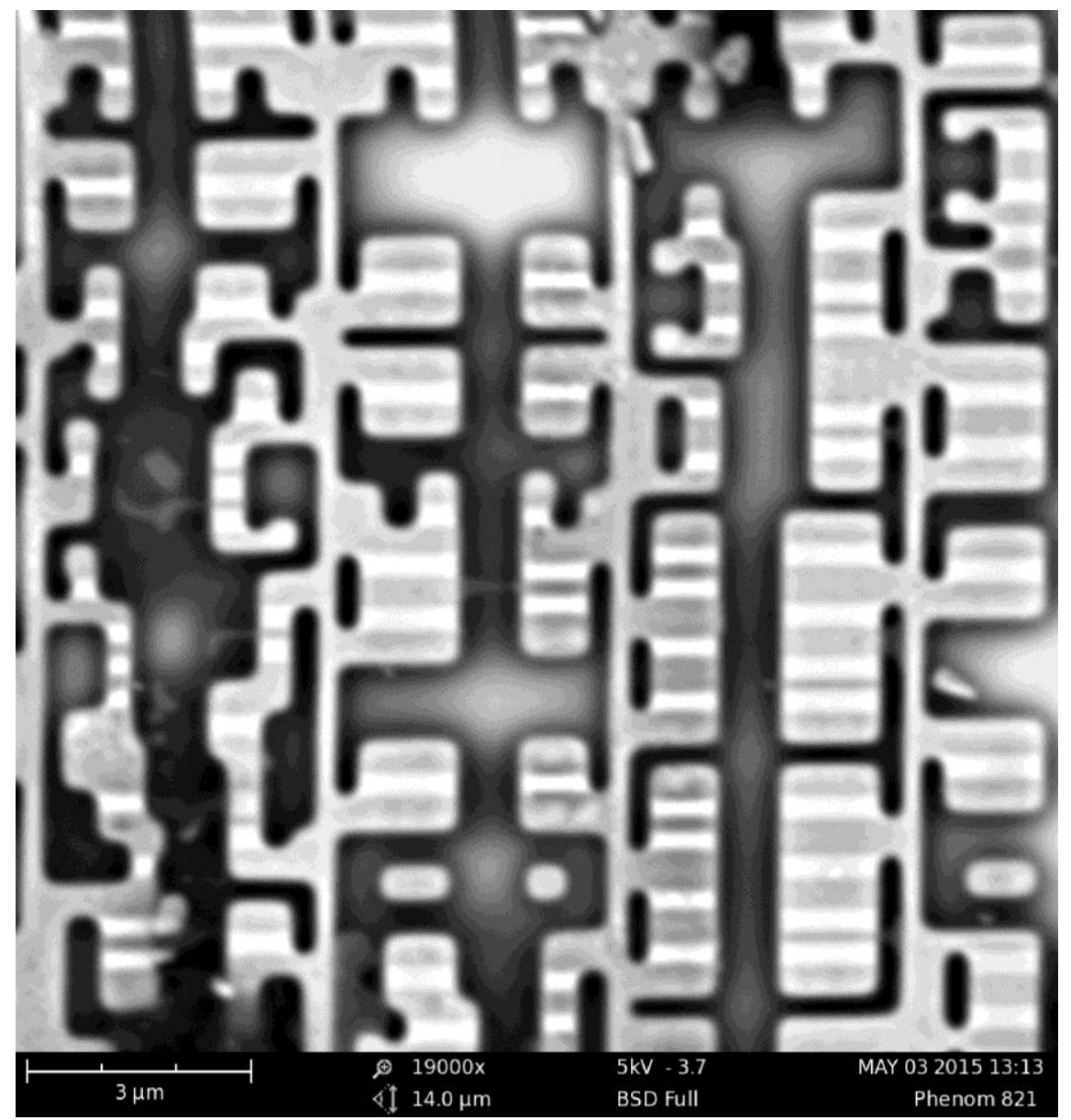

Figure 13. The Approximate Maximal Magnification Usable for Observation Provided by our Electron Microscope Phenom Pure G2 


\section{Software for Analysis}

We implemented algorithms for the first steps of image data analysis and evaluate the results for further research. We also decided to prepare basic functions including project management and uncluttered UI to provide a good foundation for the subsequent steps.

\subsection{Implementation Details}

For the last stage of the implementation (see Figure 14) we used the following tools:

- $\quad$ Qt Creator IDE 3.5.0

- Qt 5.4.2

- OpenCV 3.1.0

- Terget platforms: Windows, linux

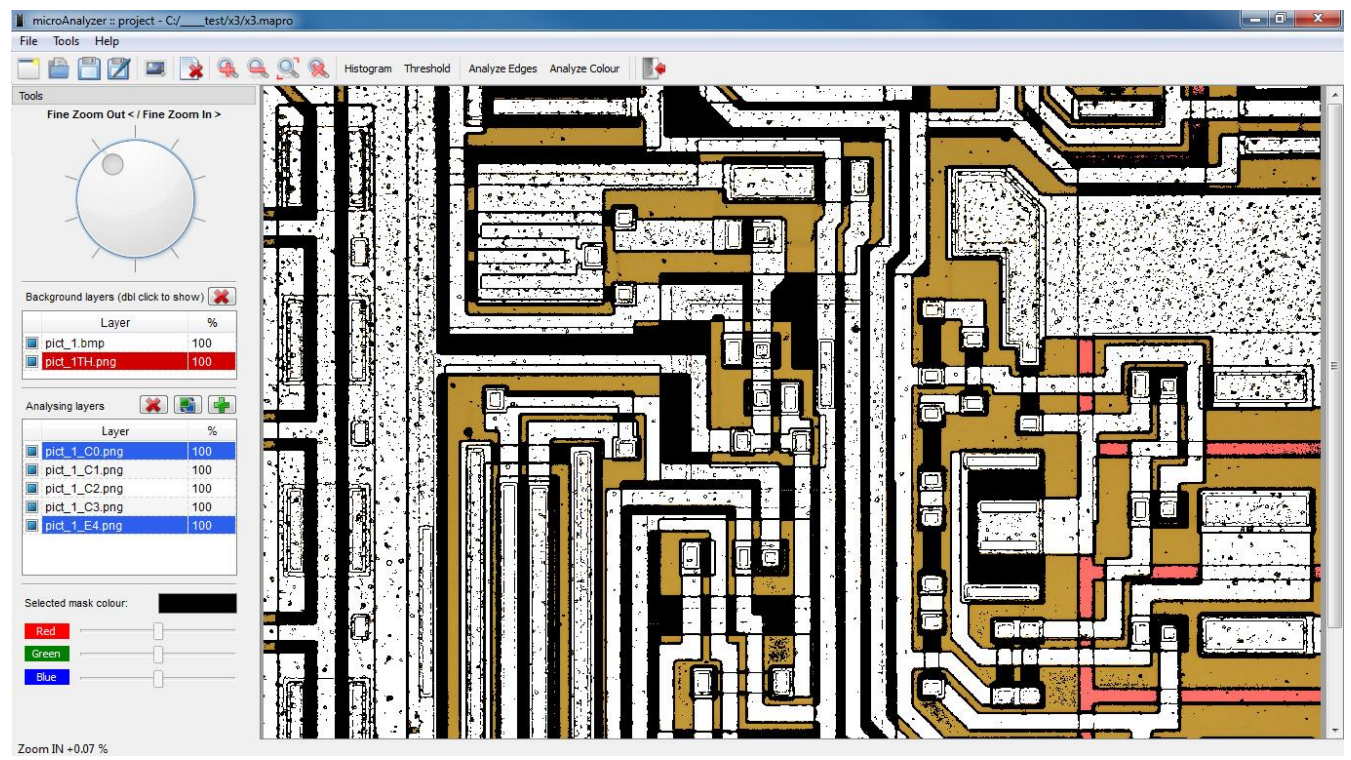

Figure 14. Screenshot of the Implemented Application

The application is able to:

- $\quad$ load and display images with following file extensions - .bmp, .tif, .png, .jpg,

- work with layers of chips - display them, set overlapping, blending,

- $\quad$ store the layers belonging to one chip into a specific project file format (extension .mapro), the project file format is based on XML standard,

- perform basic steps of analysis based on thresholding, edge analysis, color analysis and combinations of image layers.

\subsection{Threshold}

The binary thresholding function was originally intended only to help with preparation of a mask for masking out the background of the chip in an image. With a correct value, it can be easily distinguished between the chip rectangle and the surrounding. Because of the prepared mask we can easily filter out some of undesirable information, such as background of the chip might be. This application is presented in Figure 16. 
Another application where we use thresholding is the Edge analysis. Binary thresholding is a precondition for a correct use of the edge detector. Nevertheless, we will come to this usage later in the following chapter 6.3.

Mathematic description of binary thresholding is as follows:

$$
\operatorname{dst}(x, y)= \begin{cases}\operatorname{maxval} & \text { if } \operatorname{src}(x, y)>\text { thresh } \\ 0 & \text { otherwise }\end{cases}
$$

Figure 15. Mathematic Description of Binary Thresholding (source [12])

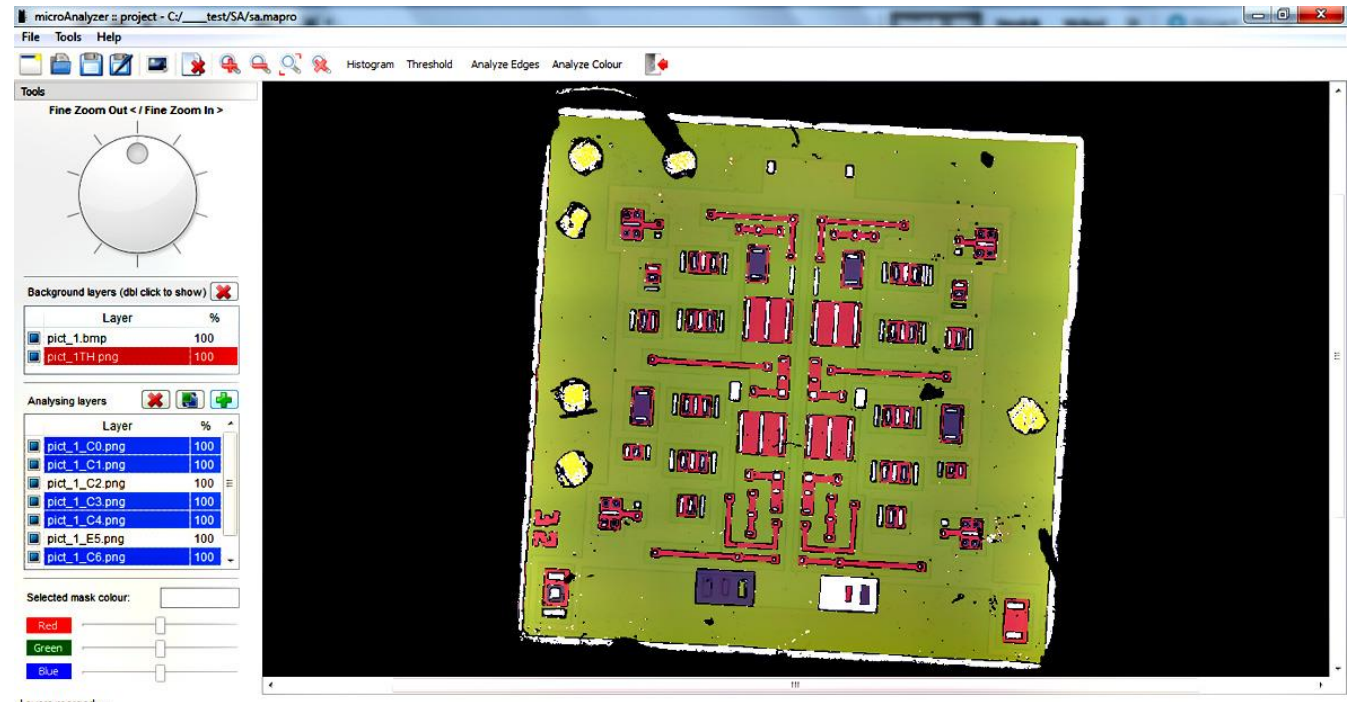

\section{Figure 16. Screenshot of the Implemented Application - Demonstration of Binary Thresholding and Color Separation}

\subsection{Edge Analysis}

For detection of edges in the bitmap image we chose Canny Edge detector, sometimes called also optimal detector. This detector is implemented in OpenCV library. The algorithm was developed by John F. Canny in 1986.

The description of the algorithm is cited from [13]:

1. Filter out any noise. The Gaussian filter is used for this purpose. An example of a Gaussian kernel of size $=5$ that might be used is shown below:

$$
\mathrm{K}=\frac{1}{159}\left[\begin{array}{ccccc}
2 & 4 & 5 & 4 & 2 \\
4 & 9 & 12 & 9 & 4 \\
5 & 12 & 15 & 12 & 5 \\
4 & 9 & 12 & 9 & 4 \\
2 & 4 & 5 & 4 & 2
\end{array}\right]
$$

2. Find the intensity gradient of the image. For this, we follow a procedure analogous to Sobel:

1. Apply a pair of convolution masks (in $\mathbf{x}$ and $\mathbf{Y}$ directions: 


$$
\begin{gathered}
\mathrm{G}_{x}=\left[\begin{array}{ccc}
-1 & 0 & +1 \\
-2 & 0 & +2 \\
-1 & 0 & +1
\end{array}\right] \\
\mathrm{G}_{y}=\left[\begin{array}{ccc}
-1 & -2 & -1 \\
0 & 0 & 0 \\
+1 & +2 & +1
\end{array}\right]
\end{gathered}
$$

2. Find the gradient strength and direction with:

$$
\begin{aligned}
& \mathrm{G}=\sqrt{\mathrm{G}_{\mathrm{x}}^{2}+\mathrm{G}_{\mathrm{y}}^{2}} \\
& \theta=\arctan \left(\frac{\mathrm{G}_{\mathrm{y}}}{\mathrm{G}_{\mathrm{x}}}\right)
\end{aligned}
$$

The direction is rounded to one of four possible angles (namely 0 , 45, 90 or 135)

3. Non-maximum suppression is applied. This removes pixels that are not considered to be part of an edge. Hence, only thin lines (candidate edges) will remain.

4. Hysteresis: The final step. Canny does use two thresholds (upper and lower):

1. If a pixel gradient is higher than the upper threshold, the pixel is accepted as an edge

2. If a pixel gradient value is below the lower threshold, then it is rejected.

3. If the pixel gradient is between the two thresholds, then it will be accepted only if it is connected to a pixel that is above the upper threshold.

Canny recommended a upper:lower ratio between 2:1 and 3:1."

\subsection{Color Analysis}

Color analysis is based on finding parts with the same or very similar color respecting a specified tolerance. This tool supplements the previously mentioned analysis algorithms. In combination with thresholding, edge detector and possibility to combine several layers with such results or partial results, we can visually process images and separate them into various layers that can be later combined again, e.g. Figure 17 and Figure 18. 


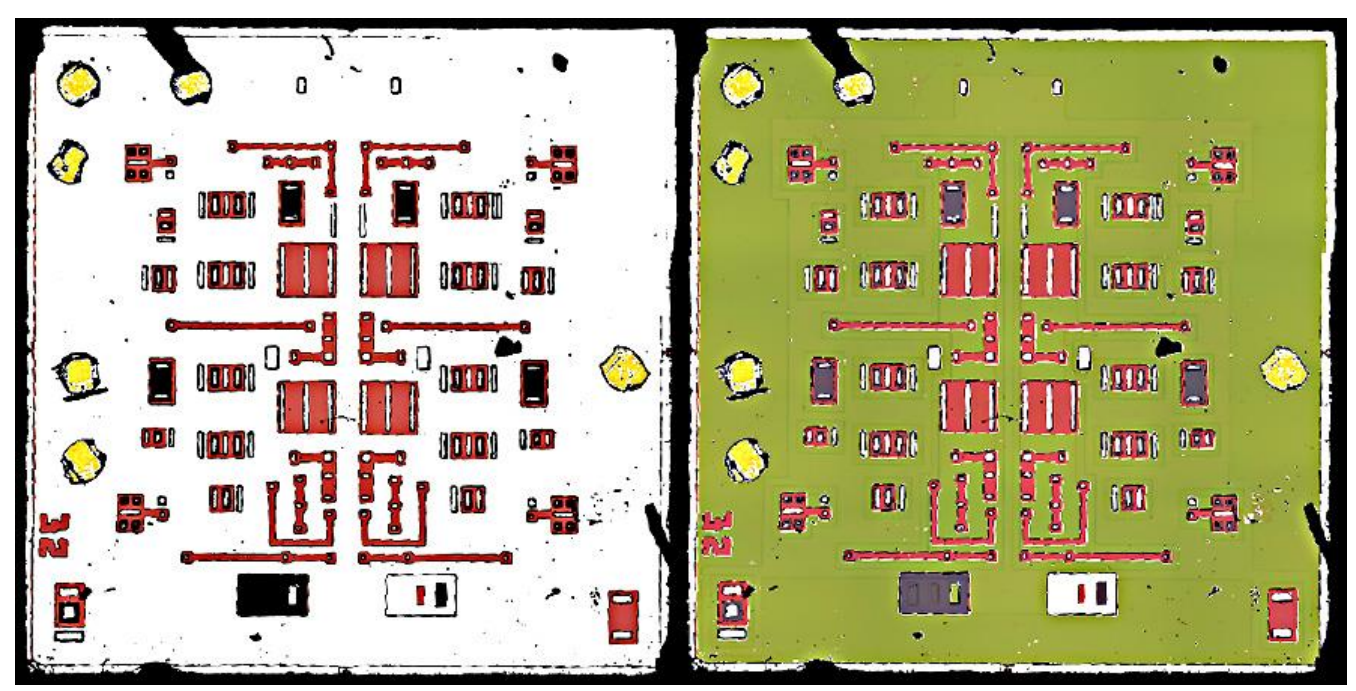

Figure 17. Screenshot of the Implemented Application - Separation of Different Layers

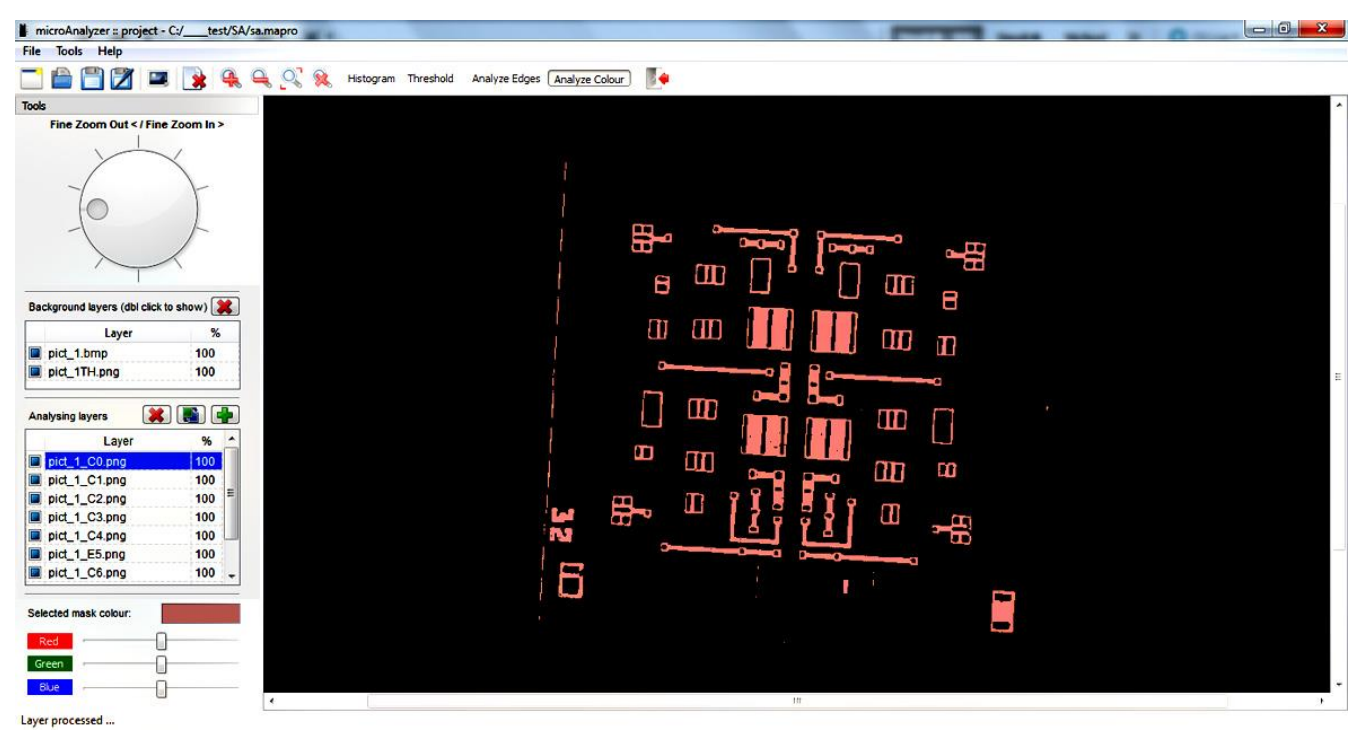

Figure 18. Screenshot of the Implemented Application - Separation of Different Layers

\section{Conclusion}

We summed up in this paper the whole process leading from obtaining chips from the plastic cards at the very beginning, to results of analysis process in the area of hardware analysis of chips with use of optical and electron microscopes. The presented software part is currently under further development, especially new approaches will be presented soon in the following papers.

\section{Acknowledgments}

This paper contains results from the project COST CZ LD14013 with the title "New solutions for multimodal biometrics - enhancement of security and reliability of biometric technologies", belonging to the ICT COST action IC1106 with the title "Integrating Biometrics and Forensics for the Digital Age".

This work was supported by The Ministry of Education, Youth and Sports of the Czech Republic from the National Programme of Sustainability (NPU II); project 
IT4Innovations excellence in science - LQ1602 and the internal Brno University of Technology project FIT-S-14-2486 "Reliability and security in IT" (CZ).

\section{References}

[1] Moore, G. E.: Cramming More Components onto Integrated Circuits. Electronics, Vol. 38, No. 8, April 1965: p. 114-117, ISSN 0018-9219, DOI: 10.1109/JPROC.1998.658762.

[2] Quality and Reliability division, Semiconductor Business Group, Professional, Device \& Solution Group, Sony Corporation: Sony Semiconductor Quality and Reliability Handbook [online]. 2011, [cit. 2013-01-10]. URL http://www.sony.net/Products/SC-HP/tec/catalog/qr.html

[3] Chernyy, N.: HOW TO: write an IC Friday post [online]. 2008, [cit. 2013-01-05]. URL http://microblog.routed.net/2008/07/15/how-to-write-an-ic-friday-post/

[4] Schobert, M.: All Chips Reversed. Die Datenschleuder, Vol. 94, 2010: pp. 17-36, ISSN 0930-1054.

[5] Torrance, R.; James, D.: The state-of-the-art in semiconductor reverse engineering. In Design Automation Conference (DAC), 2011 48th ACM/EDAC/IEEE, June 2011, ISSN 0738-100X, pp. 333338.

[6] Torrance, R.; James, D.: Reverse Engineering in the Semiconductor Industry. In Custom Integrated Circuits Conference, 2007. CICC '07. IEEE, September 2007, pp. 429-436, DOI:10.1109/CICC.2007.4405767.

[7] Kumagai, J.: Chip detectives [reverse engineering]. Spectrum, IEEE, Vol. 37, No. 11, November 2000: pp. 43-48, ISSN 0018-9235, DOI:10.1109/6.880953.

[8] Blackwell, G. R.: The Electronic Packaging Handbook. CRC Press, 1999, ISBN 978-0849385919, p. 640.

[9] Chung, D.: Materials for electronic packaging. Butterworth-Heinemann, 1995, ISBN 978-0750693141.

[10] Szendiuch, I.: Základy technologie mikroelektronických obvodů a systémů. VUTIUM, 2006, ISBN 80214-3292-6, 379 p.

[11] Tummala, R.: Fundamentals of Microsystems Packaging. McGraw Hill Professional, 2001, ISBN 9780071371698, $967 \mathrm{p}$.

[12] Miscellaneous Image Transformations - OpenCV 2.4.12.0 documentation, opencv.org [online]. 2014, [cit. 2015-12-30]. http://docs.opencv.org/2.4/modules/imgproc/doc/miscellaneous_transformations.html?highlight=thresho ld\#threshold

[13] Canny Edge Detector - OpenCV 2.4.12.0 documentation, opencv.org [online]. 2014, [cit. 2015-12-30]. http://docs.opencv.org/2.4/doc/tutorials/imgproc/imgtrans/canny_detector/canny_detector.html 
International Journal of Security and Its Applications

Vol. 10, No. 11 (2016) 